Additional Information
NOTICE: This is the author's version of a work that was accepted for publication in Construction and Building Materials. Changes resulting from the publishing process, such as peer review, editing, corrections, structural formatting, and other quality control mechanisms may not be reflected in this document. Changes may have been made to this work since it was submitted for publication. A definitive version was subsequently published in Construction and Building Materials, Vol. 40, 2013. doi: 10.1016/j.conbuildmat.2012.11.011 


\section{An Evaluation of the Suitability of SUPERPAVE and Marshall Asphalt Mix Designs as They Relate to Thailand's Climatic Conditions}

Peerapong Jitsangiam ${ }^{1 *}$, Prinya Chindaprasirt ${ }^{2}$, and Hamid Nikraz ${ }^{1}$

${ }^{1}$ Department of Civil Engineering, Curtin University, Perth, Western Australia, Australia

${ }^{2}$ Sustainable Infrastructure Research and Development Center, Department of Civil Engineering, Faculty of Engineering, Khon Kaen University, Khon Kaen 40002, Thailand

${ }^{*}$ Corresponding Author: Department of Civil Engineering, Curtin University;P.O. Box U1987, Perth, WA, Australia 6845;PH (+61) 89266 4527; FAX (+61) 89266 2681; p.jitsangiam@curtin.edu.au

\section{Abstract:}

The most commonly-used asphalt mix design in Thailand still relies on the Marshall Mix design procedure which is empirical in its nature, in the sense that it is based on data produced by experiment and observation rather than reliable "infield" data. As a result of this, the Marshall Mix design procedure has substantial drawbacks with respect to replicating the real or actual behaviour of asphalt during construction and in actual in-service conditions.

The Strategic Highway Research Program (SHRP) has developed the Superior Performance Asphalt Pavements (SUPERPAVE) mix design procedure, which shifts to a large degree away from the empiricism of the Marshall Mix design to provide a more reliable and responsive solution to actual pavement conditions.

This study aims to evaluate whether the SUPERPAVE mix design procedure can be reliably implemented under Thailand pavement conditions. A map of the Performance Grade (PG) asphalt binders was generated to cover the study area, namely the North part of Thailand, according to the SUPERPAVE asphalt classification with the highest and lowest temperature ranges that the asphalt might be subjected to. Using local materials, and considering loading and environmental conditions, a comparative study of the performance of two mixes, designed using SUPERPAVE and Marshall Mix design procedures, was carried out.

The SUPERPAVE mixes proved superior to the Marshall mixes. However, the asphalt binder commonly used in Thailand is not suitable for Thailand pavement conditions, based on the PG grade asphalt classification system.

Keywords: SUPERPAVE; Marshall; Asphalt mix design; Asphalt binders;

Performance Grade asphalt 


\section{Introduction}

\subsection{Background}

Since the fundamental knowledge of pavement engineering around the world in road and highway analysis and design was established after the World War II, the empirical approach has been relied on, resulting from a combination of real construction experience, pavement trials, and simple tests (i.e., California Bearing Ratio (CBR)). Without exception, the asphalt mix design has been used for an asphalt layer as the wearing course of a multi-layer pavement structure. The significant properties of asphalt mixes for flexible pavement surfacing are stability, durability, flexibility and skid resistance. Conventional Hot Mix Asphalt (HMA) design methods aim to determine the optimum asphalt content where an asphalt layer performs satisfactorily, particularly with respect to stability and durability [1]. There are many mix design methods used throughout the world, including the Marshall Mix design method, Hubbard-Field mix design method, Hveem mix design method and the Asphalt Institute method of mix design. Of these, only two are widely implemented, namely the Marshall Mix design method and the Hveem mix design method [2]. In Thailand, the Marshall Mix design procedure (ASTM D 1559) [3] is used for designing asphalt concrete mixes.

In Thailand, the Department of Highway (DOH) has developed specifications for highway design and construction, highway materials, and hot mix asphalt based on the Thailand National standard, which has been set up to equal the best international standards with respect to ASTM [3], and the Asphalt Institute [4]. However, in recent years, premature damage to Thailand pavements has become evident after only a short period in service. This may be attributed to higher pavement temperatures in Thailand (compared to the default standard pavement temperature usually derived from North American conditions [4]) and an increase in heavy traffic loading; the latter being brought about by high demand for road transport occurring as a result of the rapid and recent economic growth in Thailand. Furthermore, it is believed that the continuing use of the Marshall Mix design procedure for asphalt mixtures has contributed to premature pavement deterioration. Not only is the Marshall Mix procedure limited by its empirical or experimental natures, shortcomings in predictive accuracy are also evident [2]. The procedure is not able to effectively determine the full effects of crucial variables such as changes in both environmental and loading conditions. In addition, the procedure lacks reliability in producing evidence regarding the effect of the type of construction materials used (and their properties) upon pavement performance.

Recently, DOH has established a research project based on a road distress survey to identify the real causes of the premature damage occurring on recent highway construction projects [5]. The survey found that rutting is the major premature damage mechanism of Thailand's asphalt pavements, in addition to minor pavement damage through fatigue cracking. From pavement forensic analysis of pavement-layer core samples in this $\mathrm{DOH}$ study, it was concluded that the overall rutting appearing on the pavement surface results from excessive vertical deformations of the asphalt layer and the underneath base course layer of the multilayer pavement structural system. This directly indicates that the pavement structure cannot withstand the current severe pavement conditions (i.e., heavier traffic loads, 
more traffic, and higher pavement temperatures than the temperature standard [4]), as all of these conditions extend beyond the previously experienced range of conditions used in the empirical approach based on the $\mathrm{DOH}$ design standard with the standard axle load of dual wheel carrying $80 \mathrm{kN}$ load [11] and the default asphalt test temperature of $25^{\circ} \mathrm{C}$ [3]. Consequently, $\mathrm{DOH}$ has established a task to investigate alternative methods to improve pavement material performance. The Thailand HMA relying on the Marshall mix design [4] needs to be improved in accordance with the SUPERPAVE mix design protocol $[6,7]$.

It is widely believed that the Marshall Mix design is not appropriate in application for mixes with a high degree of shear susceptibility [1]. For example, the simple compaction method, in accordance with the Marshall Mix protocol, does not effectively simulate the densification that occurs in real pavement under traffic loading because of a lack of sensible laboratory compaction to replicate the real compaction in the field [8]. Due to these and other drawbacks, the procedure was withdrawn from the American Standard Testing Procedures in 1998 [9].

Due to the above outcome, many countries still relying on the Marshall Mix design have been urged to adopt more accurate and up-to-date mix design and evaluation procedures [1]. Recent research and development efforts in the Strategic Highway Research Program (SHRP) have focused on the establishment of performancebased asphalt binder and asphalt mix specifications [8, 10].

The main objective of the SHRP Asphalt Program has been to develop a mixture design method that incorporates a performance-based asphalt binder specification and an accelerated performance-based test. The product designed by the new mixture design system is now known as SUPERPAVE (SUperior PERformance PAVEments) [7, 8].

The special task of the $\mathrm{DOH}$ project, conducted in collaboration with Thai universities, was to carry out preliminary research on the properties and performance of asphalt mixed aggregates, adhering to SUPERPAVE mix design procedures. Subsequent to this, the DOH performed further tests and produced a summary of all test results for all asphalts and aggregates in Thailand. All the tests were performed following $\mathrm{DOH}$ testing protocols, with some supplementary tests. However, the $\mathrm{DOH}$ projects still need further support and development in order to enhance current knowledge and to create new knowledge around the implementation of SUPERPAVE in Thailand.

Aspects of the research project [11] have already been detailed. Some results in this paper were funded by the Thailand Research Fund (TRF) through the "TRF Research Career Development Grant (2008-2010)", a scheme which aims to support researchers, strengthen institutions and motivate universities or host institutions to increase their investment in supporting research. This paper presents part of the above research project which proposes an appropriate asphalt mix design and selection of asphalt binder type to suit Thailand's climatic conditions.

\subsection{Project aims and scope}

The main aims of this research are to produce and promote a successful and reliable asphalt mix design of HMA for application in the construction of Thailand's road pavement, in addition to reporting the recent key findings of other studies in the SUPERPAVE approach for Thailand. This design will replace current inadequate designs and should eliminate associated instability attributable to asphalt binder 
characteristics, unsuitable aggregate gradation, ineffective compaction methods, and unreliable performance tests.

The project should lead to an improved HMA mix design and result in a costeffective and highly reliable design. To achieve the main aims, the research-specific aims are to:

- Characterise commonly-used asphalt binders in Thailand with respect to the Performance Grade (PG-grade) system of the SUPERPAVE mix design.

- Construct a pavement temperature map of North Thailand (termed "the study area") for asphalt binder selection.

- Introduce the SUPERPAVE mix design to Thailand, based on the study area results.

- Compare the performance of asphalt samples produced from the SUPERPAVE and Marshall mixes.

This research project used the North part of Thailand as the study area, comprising 15 provinces: Chiang Mai, Chiang Rai, Kamphaeng Phet, Lampang, Lamphun, Mae Hong Son, Nan, Pha Yao, Phetchabun, Phichit, Phitsanulok, Phrae, Sukhothai, Tak, and Uttaradit.

\subsection{Current situation of asphalt binder testing in Thailand}

The current tests used to characterise asphalt binders in Thailand still rely on traditional testing methods, and these attempt to identify only the physical properties (i.e., penetration, viscosity and ductility) of asphalt binders. Generally, these tests are performed at a default standard test temperature. The results of these tests are usually used to evaluate whether the material complies with the specification criteria. However, there are significant limitations regarding the tests and these can be summarised as follows:

- The most current standard tests are still empirical (i.e., based on data produced from experiment and observation) and therefore the test results per se cannot fully represent or replicate the actual performance of pavement in situ.

- The tests exhibit shortcomings with regard to demonstrating the actual material properties of pavement. For example, in the field, pavement is exposed to a wide range of temperatures and the property of viscosity is only evident at high temperatures. As the traditional tests currently in use only employ a default standard test temperature, viscosity cannot be accurately measured.

- The current asphalt classification system cannot distinguish between performances of a number of asphalt binders within the same asphalt group. The system only demonstrates the range in similarity of the physical properties of the material. 
- These properties can only be realistically evaluated, and differences in performance demonstrated, under exposure to a wide range of temperature conditions, as found in the field.

\subsection{The SUPERPAVE mix design}

In 1987, due to the drawbacks found in both asphalt binder and mix specifications, the US congress supported a five year research program of the Strategic Highway Research Program (SHRP) to improve the performance and durability of US roads and to enhance the safety of motorists. Some of the research funds were used for the development of performance-based asphalt specifications which would correspond more directly to those based on laboratory analysis and field performance [12]. A bi-modal grading system, based on rational performance indices, was established for both low temperature and high temperature pavement service.

The SHRP established SUPERPAVE to greatly improve the performance of asphalt under severe conditions such as temperature variations, traffic growth and changing environments. The SUPERPAVE design system consists of three interrelated parts:

1. A performance graded (PG) asphalt binder specification, and tests based on the pavement temperature range of a design area.

2. A mixture design system which employs both a volumetric mixture design with a SUPERPAVE gyratory compactor (SGC), and an analysis/performance prediction element. An SGC $\left(1.25^{\circ}, 30\right.$ gyrations/min and $0.6 \mathrm{MPa}$ ram pressure for a $150 \mathrm{~mm}$ mould) is used for the evaluation of volumetric properties and strength of compacted mixes [13].

3. An evaluation of the asphalt sample(s) performance.

\subsubsection{Performance Graded asphalt binder specification (PG Grade)}

SUPERPAVE uses an improved system for testing, specifying, and selecting asphalt. The physical property requirements within the specifications are constant among all PG grades. What differentiates the various binder grades is the temperature at which the requirements must be met. For example, a binder classified as PG 64-22 means the binder must meet high temperature physical property requirements up to a temperature of at least $64^{\circ} \mathrm{C}$; low temperature physical properties must be met down to at least $-22^{\circ} \mathrm{C}$. These physical properties are directly related to field performance, thus the greater the first (high) temperature value is, the more resistant the binder should be to high temperature distresses such as rutting or shoving. Likewise, the lower the second (low) PG temperature value is, the more resistant the binder should be to low temperature cracking. The high and low temperature designations extend in both directions as far as necessary, in sixdegree increments, making the number of possible grades almost unlimited. The 
more common paving grades used in the US are PG 64-22, PG 70-22, PG 76-22, PG58-22, PG 64-28, PG 58-28, and PG 52-34 [7].

The temperature analysis for the PG grade of asphalt binders in the US was based on a database of temperature information from over 7,000 weather stations in the US and Canada, to allow users to select binder grades for the climate at a particular project location [7]. Based on the SUPERPAVE temperature database standard [16], for each year that the weather stations had been in operation, the hottest seven-day period was identified, and the average maximum air temperature of this seven-day period was calculated and for all the years of operation, the mean and standard deviation of the seven-day average maximum air temperature was calculated. Similarly, the one-day minimum air temperature of each year was identified, and the mean and standard deviation were calculated. Weather stations with less than 20 years of data were not used.

As the design temperatures used for selecting asphalt binder grades are based on pavement temperatures, the air temperatures from the weather stations' databases must be converted into pavement temperatures. For surface layers, SUPERPAVE specifies the locations for the high pavement design temperature to be at a depth 20 $\mathrm{mm}$ below the pavement surface, and the low pavement design temperature to be taken at the pavement surface. A simulation of asphalt deterioration during construction and service conditions is required before characterisation of asphalt performances can be carried out, and this is done by using new test protocols e.g., Rolling Thin Film Oven Test, Pressure Ageing Vessel Test and the Modulus test using a dynamic shear rheometer. For certain PG grades, variation of testing temperatures during the tests is used, based on the design location.

\subsubsection{Main issues of SUPERPAVE mix design}

The SUPERPAVE design mainly uses volumetric properties for the mix design, similar to the Marshall method. The design air void is approximately $4 \%$. The main differences between these two methods are as follows:

- Asphalt specimens for SUPERPAVE were prepared using the gyratory compactor with kneading actions which can reliably replicate the roller compaction in the filed instead of the Marshall compactor which can provide only the static compaction.

- The gyratory compaction level varied with design traffic and the average maximum temperature in a design location.

\subsubsection{Testing requirements}

Asphalt samples were prepared and subjected to performance tests e.g., moisture sensitivity, indirect tensile strength, resilient modulus, permanent deformation and fatigue performance. Testing requirements were classified at three levels, based on design traffic volume:

- Volumetric Mix Design for traffic $10^{6}$ Equivalent Standard Axle Loads (ESALs).

- Intermediate Analysis for traffic $10^{7}$ ESALs.

- Complete Analysis for traffic greater than $10^{7}$ ESALs. 
The SUPERPAVE specifications cover the selection and evaluation of proper asphalt binders in accordance with the PG grade system, specifications and testing method for aggregate, and appropriate asphalt mix design procedures. Restricted zones for aggregate gradation which might lead to rutting are indicated in the specifications. The asphalt mix was designed based on the volumetric properties of asphalt specimens compacted by the gyratory compactor.

In this research, a performance grading map showing the required asphalt binder grades for the different provinces in the study area was generated. Representative aggregate and asphalt samples were collected and proposed. A comprehensive evaluation of the collected materials was carried out to ensure that these materials conformed to the SUPERPAVE mix design procedures, and that area-specific conditions of traffic and environment were taken into consideration.

Marshall and SUPERPAVE mix design procedures were carried out using the collected asphalt binders and aggregate samples. Comparison between the two mix design procedures included optimal asphalt content, aggregate gradation, and the mixes' mechanical performance. The mechanical performance testing and evaluation consisted of Marshall stability, loss of Marshall stability, indirect tensile strength, loss of indirect tensile strength, resilient modulus, and creep performance.

\section{Experimental program and research methodology}

\subsection{Material characteristics}

The two main materials of HMA mix design - aggregates and asphalt binders, were collected and characterised according to the DOH testing procedure [14]. This process was carried out according to the Thailand national standard for asphalt binder characteristics and the SUPERPAVE recommended evaluation tests.

\subsubsection{Asphalt binders}

The asphalt binders of types AC60-70, in accordance with the DOH specifications [15], were selected for this study because they are the most commonly used asphalt binders in Thailand for normal HMA mixes, along with the high quality HMA mix of AC60-70 and Polymer Modified Asphalt (PMA), respectively. Samples of these asphalt binder types were collected from the asphalt refinery plant in Bangkok, Thailand. Preliminary evaluation of the collected asphalt samples was conducted to assure that their significant properties complied with the DOH specifications for fresh binders and treated binders after Thin Film Oven (TFO) treatment, which accelerates the asphalt binder's ageing process. The evaluation comprised of flash point, rotational viscosity at $135^{\circ} \mathrm{C}$, penetration at $25^{\circ} \mathrm{C}$, specific gravity at $25^{\circ} \mathrm{C}$, ductility at $25^{\circ} \mathrm{C}$, softening point, penetration, and weight loss on heating. After the national specifications of asphalt binders were met, the selected asphalt binders were then characterised following the SUPERPAVE protocol, with Dynamic Shear Rheometer (DSR) testing of fresh samples and aged samples, after Rolling Thin Film Oven (RTFO) treatment and the Pressure Ageing Vessel (PAV) test, at different targeted temperatures which were derived from the air temperature analysis.

\subsubsection{Aggregates}


The aggregate in this study was crushed limestone obtained from the Phitsanulok province, to be used as the aggregate representative for the entire study. Physical properties of the aggregate sample were evaluated. These evaluations included coarse and flat/elongated particles, angularity, sand equivalency, coarse and fine aggregate specific gravity and absorption, and Los Angeles abrasion. The selected aggregate gradation was carried out in accordance with $\mathrm{DOH}$ specifications [14]. The recommended gradation for the national HMA mix design relied on the Marshall Mix design protocol.

\subsection{Temperature analysis}

Minimum and seven-day consecutive maximum air temperature data from different weather stations located in every province in the North part of Thailand (the study area) was collected from the Northern Meteorological Centre, Chiang Mai, Thailand, where all weather data for the targeted provinces was kept. A set of the minimum and seven-day consecutive maximum air temperatures from 2000 to 2010 was collected for the temperature-based analysis used in this study. The collected air temperature data was converted into pavement temperatures (in order to select asphalt binder grades), and these were analysed to generate the temperature zoning map for the study area. Long Term Pavement Performance Program [LTPP] Bind software [16] was employed to convert the air temperatures into pavement temperatures for this study. For the surface layers, the SUPERPAVE design defines the location for high pavement design temperature at a depth $20 \mathrm{~mm}$ below the pavement surface, and the low pavement design temperature to be taken at the pavement surface [7].

\subsection{HMA mix design}

The Marshall Mix design (ASTM D1559) [3, 17] and SUPERPAVE mix design (AASHTO TP4) [7] protocols were used for the HMA mix design, based on the use of local Thai asphalt binders and aggregates. For both mixes, The DOH specifications [14], which are the recommended gradations for wearing courses, were followed in optimising the aggregate grading characteristics. These traditional aggregate gradation characteristics of HMA design were evaluated as to whether they complied with the suggested range of aggregate grading characteristics according to the SUPERPAVE gradation.

\subsection{Performance tests}

Two sets of samples were designed to make a comparison between the performance of asphalt samples, where the asphalt contents were derived from either the Marshall Mix design procedure or the SUPERPAVE procedure. All test samples were prepared based on the same compaction procedure of the gyratory compactor at a $4 \%$ targeted air void to maintain the similar densification of test samples. The first set of samples was compacted using the designed gradation characteristics following the $\mathrm{DOH}$ specification recommended gradation [14]. The samples were compacted at the optimum asphalt content obtained from the Marshall Mix design procedure. The second set was compacted using the optimal gradation and asphalt content obtained from the SUPERPAVE design procedure. The 
performance of both mixes was evaluated in terms of Marshall stability, loss of Marshall stability, indirect tensile strength, loss of indirect tensile strength, resilient modulus, and dynamic creep.

\section{Results and discussion}

\subsection{Characterising the materials used in this study}

Table 1 lists the asphalt binder classification tests and the results of the tests performed. The results of the tests indicate that the tested asphalt binder can be graded as AC60-70 penetration asphalt according to AASHTO M 20 and DOH specifications. To find the performance grade of the used asphalt binder according to SHRP binder performance specification (AASHTO MP1) [8], DSR testing of fresh and treated samples was conducted at different test temperatures, (after the RTFO and PAV processes). It was found that the performance grade of the asphalt was $P G$ 64-22 (Table 1), which is the AC60-70 of a penetration grade for a commonly used asphalt binder in Thailand. Figures 1 (a) and 1 (b) show the DSR test results, which are used for the estimation of the lower-upper temperature range according to the binder performance grade specification. This asphalt met both the high temperature property requirements, at least up to a temperature of $64^{\circ} \mathrm{C}$, and low temperature physical property requirements to at least $-22^{\circ} \mathrm{C}[7]$.

Table 1.

Properties of the asphalt binder used in the study

\begin{tabular}{lcc}
\hline Tests & Criteria & Test results \\
\hline Flash point & $230^{\circ} \mathrm{C} \mathrm{min}{ }^{*}$. & 312 \\
Rotational viscosity at $135^{\circ} \mathrm{C}$ & $3 \mathrm{~Pa}$.s max & 0.678 \\
Penetration & $60-70$ & 65 \\
Specific Gravity at $25^{\circ} \mathrm{C}$ & $1.01-1.06$ & 1.016 \\
Ductility at $25^{\circ} \mathrm{C}$ & $100 \mathrm{~min}$ & 138 \\
Softening point, ${ }^{\circ} \mathrm{C}$ & $48-56$ & 52 \\
Penetration of residue, $\%$ original & $54 \mathrm{~min}$ & 63 \\
$\mathrm{Weight}^{*}$ loss on heating, \% & $0.8 \mathrm{max}$ & 0.46 \\
$\mathrm{G}^{*} / \sin \delta$ at $64^{\circ} \mathrm{C}($ fresh), $\mathrm{kPa}$ & $1.0 \mathrm{~min}$ & 1.195 \\
$\mathrm{G}^{*} / \sin \delta$ at $64^{\circ} \mathrm{C}(\mathrm{RTFO}), \mathrm{kPa}$ & $2.2 \mathrm{~min}$ & 2.631 \\
$\mathrm{G}^{*} / \sin \delta(\mathrm{PAV}), \mathrm{MPa}$ & $5.0 \mathrm{max}$ & $5.0^{* *}$ \\
\hline
\end{tabular}

${ }^{*}$ min=minimum; max=maximum

** $5.0 \mathrm{MPa}$ of $\mathrm{G}^{*} / \sin \delta(\mathrm{PAV})$ at $22^{\circ} \mathrm{C}$ 


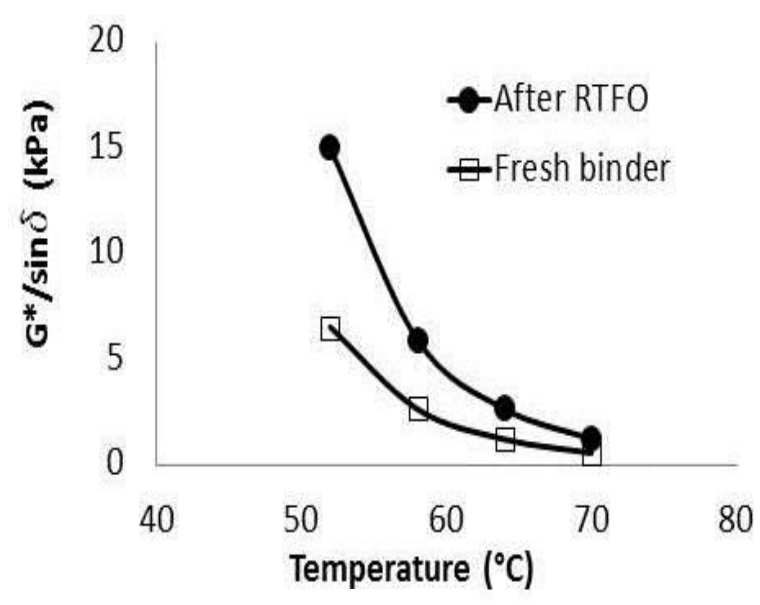

(a)

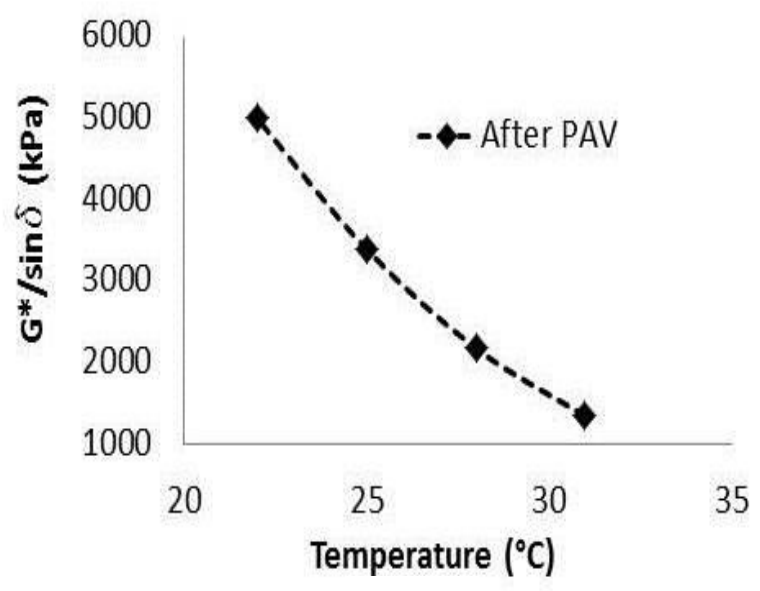

(b)

Figure 1. Dynamic shear rheometer testing of fresh and treated asphalt binders

SUPERPAVE specifications for aggregate properties are based on both consensus and source properties. Consensus properties comprise coarse aggregate angularity, fine aggregate angularity, flat and elongated particles and clay content. Consensus property levels of acceptance depend on traffic level and the depth of the layer below the surface. Source properties include toughness, soundness, and how deleterious the materials are, and they depend upon the source specification limits (Asphalt Institute 2001).

Table 2 shows the aggregate properties found in this study. The results show that the used aggregate meets both the consensus properties and source properties of the DOH specification [14].

Table 2.

Properties of the aggregate used in the study

\begin{tabular}{lcc}
\hline Tests & Criteria & Test results \\
\hline Coarse aggregate angularity & $90 / 100 \% \min ^{*}$ & $100 / 100 \%$ \\
Fine aggregate angularity & $45 \% \min$ & $46 \%$ \\
Flakiness index, \% & $35 \% \max ^{*}$ & $43 \%$ \\
Elongation index, $\%$ & $5 \% \min$ & $12 \%$ \\
Sand equivalent & $45 \mathrm{~min}$ & 65 \\
Coarse aggregate specific gravity & $\mathrm{n} / \mathrm{a}$ & 2.438 \\
Coarse aggregate absorption & $\mathrm{n} / \mathrm{a}$ & $1.3 \%$ \\
Fine aggregate specific gravity & $\mathrm{n} / \mathrm{a}$ & 2.506 \\
Fine aggregate absorption & $\mathrm{n} / \mathrm{a}$ & $4 \%$ \\
Mixed aggregate specific gravity & $\mathrm{n} / \mathrm{a}$ & 2.483 \\
Abrasion lost & $40 \%$ max & $23 \%$ \\
Abrasion ratio & $\%$ & $16 \%$ \\
\hline
\end{tabular}

${ }^{*}$ min=minimum; max=maximum

${ }^{* *}$ Abrasion loss was determined at the 500 revolutions of the abrasion test

${ }^{* * *}$ Abrasion ration was determined from the abrasion loss ratio of 100 revolutions to 500 revolutions of the abrasion tests 


\subsection{Temperature zone of the study area}

When using SUPERPAVE specifications of PG grade, pavement temperature becomes a major factor for grading an asphalt binder, and comprehensive weather investigation, which has been conducted in the US and Canada (where SUPERPAVE is widely used) is essential. However, new countries that wish to adopt the SUPERPAVE mix design have to investigate and construct temperature data for the PG grade of asphalt binders used in a particular construction area.

In this study, the weather data from 15 weather stations distributed across every province of the North part of Thailand (the study area) was collected. Collected data covered only 11 years (from 2000 to 2010) of continuous temperature recording available in the study area. This did not meet the SUPERPAVE requirements, in that 20 years of temperature data is required. However, this was all the data available from the study area. The gathered air temperature data was analysed to obtain the yearly minimum recorded air temperature and the yearly average consecutive sevenday maximum air temperature, in addition to recording the standard deviations of both temperatures.

Figure 2 exhibits the temperature zones of the study area in accordance with the PG grade of light traffic (less than 10 million ESALs). It was found that two asphalt grades were required for the study area. PG 70-10 was found to be suitable for most areas of the study area, and only Phichit province required the asphalt to be of $P G$ 64-10 grade. Selected high temperature asphalt grades had to be shifted one or two grades up for slow or standing loads [4]. In addition, high temperature grades had to be shifted up in case of extraordinarily high numbers of heavy traffic loads (higher than 30 million ESALs) [7].

It is of significant note that since the commonly used asphalt binder grade in Thailand of AC60-70 was classified as PG 64-22 in accordance with the performance test results of the SHRP binder performance specification, as shown in Section 3.1, it cannot be used in all parts of the study area, except Phichit where PG 64-10 was preferable (see Figure 2). In these areas, the new required asphalt binder has to be modified to shift its grade to PG 70-10. In steep climbing lanes, those areas where heavy trucks reduce speed, the required asphalt grade needed to be adjusted by one extra grade. Therefore, the required grades were of PG 76-10 and PG 82-10 for medium (10 million - 30 million ESALs) and high (more than 30 million ESALs) traffic. To reach to these grades, the commonly used asphalt binder in Thailand needs to be modified using polymers or other modifier additives to improve its existing properties. 


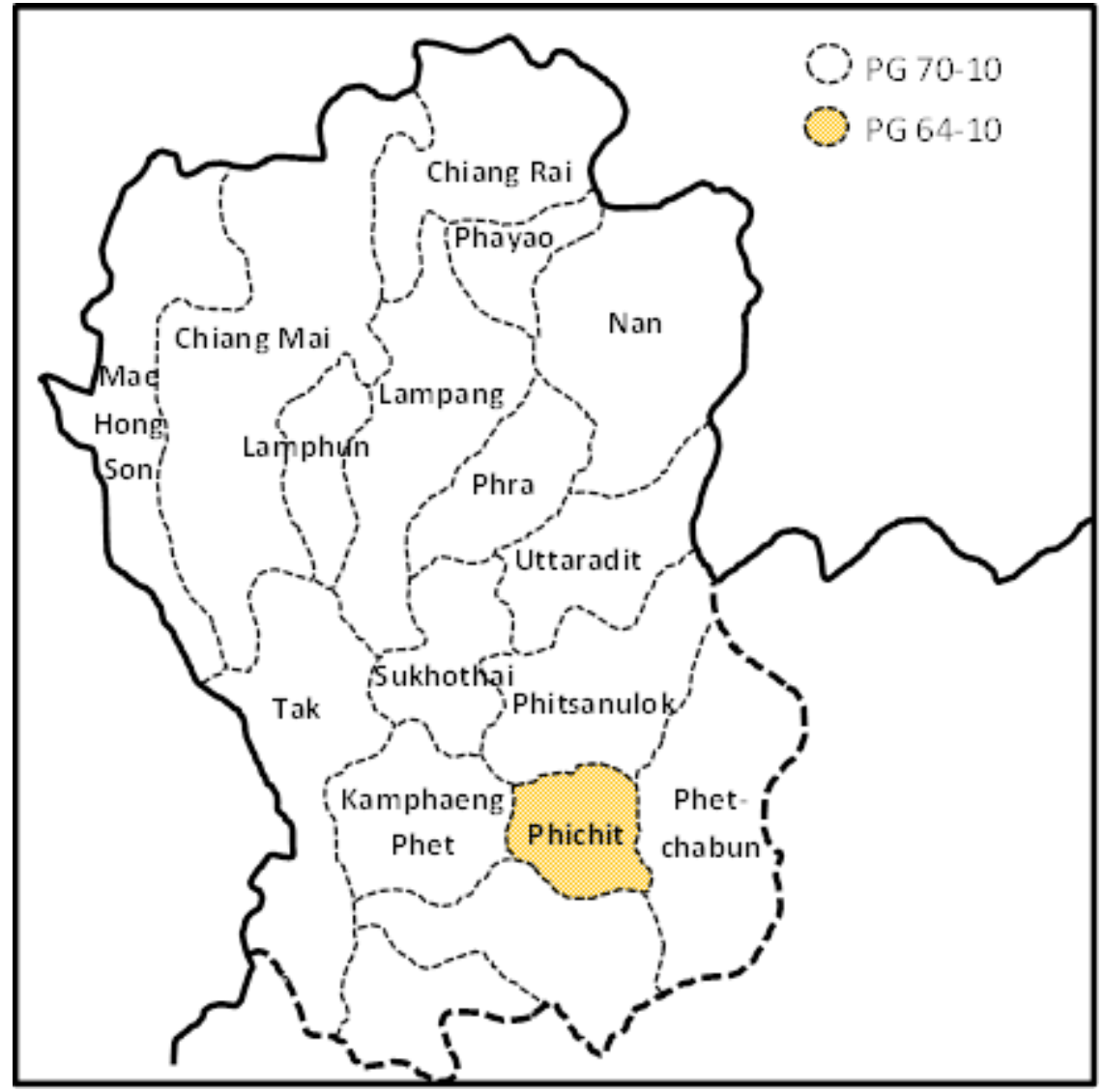

Figure 2. Temperature zone of asphalt binder selection for the study area 
Table 3.

Temperature investigation of the study area

\begin{tabular}{|c|c|c|c|c|c|c|c|c|c|c|c|c|c|c|}
\hline \multirow[t]{3}{*}{ Province } & \multirow[t]{3}{*}{ Latitude } & \multirow{2}{*}{\multicolumn{4}{|c|}{ Air Temperature $\left({ }^{\circ} \mathrm{C}\right)$}} & \multicolumn{4}{|c|}{$\begin{array}{l}95 \% \text { Reliability of Max-Min } \\
\text { values }\end{array}$} & \multirow{2}{*}{\multicolumn{2}{|c|}{$\begin{array}{l}\text { Pavement } \\
\text { Temperature }\end{array}$}} & \multirow{2}{*}{\multicolumn{3}{|c|}{$\begin{array}{c}\text { Performance Grade (PG) } \\
\text { (by traffic) } \\
\text { (Million ESALs }^{*} \text { ) }\end{array}$}} \\
\hline & & & & & & \multicolumn{2}{|c|}{ Max. Temp } & \multicolumn{2}{|c|}{ Min. Temp } & & & & & \\
\hline & & Max & ${ }^{* \star} S D_{\text {airmax }}$ & Min & ${ }^{\star \star} \mathrm{SD}_{\text {airmin }}$ & $\begin{array}{l}\text { Lower } \\
\text { Bound }\end{array}$ & $\begin{array}{l}\text { Upper } \\
\text { Bound }\end{array}$ & $\begin{array}{l}\text { Lower } \\
\text { Bound }\end{array}$ & $\begin{array}{l}\text { Upper } \\
\text { Bound }\end{array}$ & Max & Min & $<10$ & $10-30$ & $>30$ \\
\hline Chiang Rai & $19^{\circ} 55^{\prime}$ & 36.9 & 0.645 & 8.0 & 2.320 & 36.5 & 37.4 & 6.5 & 9.6 & 64.9 & 8.0 & $70-10$ & $76-10$ & $82-10$ \\
\hline Mae Hong Son & $19^{\circ} 16^{\prime}$ & 40.4 & 1.241 & 8.5 & 1.846 & 39.5 & 41.2 & 7.3 & 9.7 & 68.2 & 8.5 & $70-10$ & $76-10$ & $82-10$ \\
\hline PhaYao & $19^{\circ} 10^{\prime}$ & 37.9 & 0.724 & 8.6 & 2.355 & 37.5 & 38.5 & 7.0 & 10.2 & 65.6 & 8.6 & $70-10$ & $76-10$ & $82-10$ \\
\hline Chiang Mai & $18^{\circ} 48^{\prime}$ & 38.5 & 1.088 & 8.5 & 2.658 & 37.9 & 39.3 & 6.8 & 10.3 & 66.2 & 8.5 & $70-10$ & $76-10$ & $82-10$ \\
\hline Nan & $18^{\circ} 47^{\prime}$ & 39.4 & 0.777 & 7.5 & 2.486 & 38.9 & 39.9 & 5.8 & 9.1 & 66.8 & 7.5 & $70-10$ & $76-10$ & $82-10$ \\
\hline Lamphun & $18^{\circ} 30^{\prime}$ & 39.8 & 1.149 & 9.5 & 2.124 & 39.1 & 40.6 & 8.1 & 10.9 & 67.3 & 9.5 & $70-10$ & $76-10$ & $82-10$ \\
\hline Lampang & $18^{\circ} 17^{\prime}$ & 40.7 & 0.600 & 9.5 & 2.248 & 40.3 & 41.1 & 8.0 & 11.1 & 67.7 & 9.5 & $70-10$ & $76-10$ & $82-10$ \\
\hline Phrae & $18^{\circ} 09^{\prime}$ & 39.3 & 0.683 & 10.6 & 2.184 & 28.9 & 39.8 & 9.1 & 12.0 & 66.4 & 10.6 & $70-10$ & $76-10$ & $82-10$ \\
\hline Uttaradit & $17^{\circ} 38^{\prime}$ & 39.3 & 0.663 & 12.9 & 2.045 & 38.8 & 39.7 & 11.6 & 14.4 & 66.1 & 12.9 & $70-10$ & $76-10$ & $82-10$ \\
\hline Sukhothai & $17^{\circ} 01^{\prime}$ & 39.6 & 0.823 & 12.1 & 1.838 & 39.1 & 40.2 & 10.8 & 13.3 & 66.3 & 12.1 & $70-10$ & $76-10$ & $82-10$ \\
\hline Phichit & $16^{\circ} 26^{\prime}$ & 34.4 & 1.148 & 12.1 & 4.306 & 33.8 & 35.6 & 9.2 & 14.9 & 61.7 & 12.1 & 64-10 & $70-10$ & $76-10$ \\
\hline Tak & $16^{\circ} 53^{\prime}$ & 40.9 & 1.07 & 5.9 & 2.279 & 40.2 & 41.7 & 4.4 & 7.6 & 67.7 & 5.9 & $70-10$ & $76-10$ & $82-10$ \\
\hline Phitsanulok & $16^{\circ} 49^{\prime}$ & 38.6 & 0.698 & 12.7 & 1.709 & 38.1 & 39.0 & 11.5 & 13.8 & 65.1 & 12.7 & $70-10$ & $76-10$ & $82-10$ \\
\hline Phetchabun & $16^{\circ} 25^{\prime}$ & 39.4 & 0.750 & 10.7 & 2.201 & 38.9 & 39.9 & 9.2 & 12.2 & 65.8 & 10.7 & $70-10$ & $76-10$ & $82-10$ \\
\hline KamphaengPhet & $16^{\circ} 28^{\prime}$ & 38.9 & 0.555 & 13.2 & 0.555 & 38.6 & 39.4 & 11.9 & 14.6 & 65.3 & 13.2 & $70-10$ & $76-10$ & $82-10$ \\
\hline
\end{tabular}

*ESALs $=$ Equivalent single axial loads; $;{ }^{*} \mathrm{SD}=$ Standard deviation 
Table 3 shows the calculated average air temperatures and standard deviations from all provinces in the study area. In addition, Table 3 shows the calculated pavement high and low temperatures to a factor of $95 \%$ reliability. A $95 \%$ reliability level was used in this conversion (Table 3). Reliability is the probability in a single year that the actual temperature (one day low or seven day high) will not exceed the design temperature. A higher reliability demonstrates a lower risk. The selection of degree of reliability depends on road class, traffic level, binder cost and availability [7].

\subsection{Marshall Mix design (ASTM D1559)}

The Marshall asphalt concrete mix design procedure is the most commonly used asphalt mix design procedure in Thailand, (ASTMD1559 for four inch diameter samples). The DOH aggregate grading specification is recommended to determine the optimum mixed aggregate characteristics for the Marshall design used in Thailand [14]. The Marshall Mix design procedure was the method used for determining the optimum asphalt content. The optimum asphalt content selected resulted from several trial mixes, providing targeted air voids of a compacted standard sample at $4 \%$. In this study, the optimum asphalt content was $5.20 \%$ of total mix by mass. At the obtained optimum content, Marshall Stability, flow, voids filled with asphalt, and voids in mineral aggregate values were determined.

\subsection{SUPERPAVE mix design (AASHTO TP4)}

SUPERPAVE uses volumetric analysis for the mix design within the three main steps in the testing and analysis processes. The selection of a design aggregate structure is performed and the optimum asphalt content is chosen for the selected structure. The evaluation of moisture sensitivity of the design mixture is last.

Due to the gyratory compactor being introduced to the SUPERPAVE mix design, the number of gyratory compactor gyrations needs to be specified to compact the asphalt test sample(s) of trial mixes. The number of gyrations depended upon both average design high air temperature and design ESALs. In this study, a traffic level of between 10 and 30 million ESALs was selected to represent the medium traffic which is the traffic condition of the most road network in Thailand, within the range of a warm air temperature of the overall study area of $43-44^{\circ} \mathrm{C}$. Consequently, the recommended number of gyrations are $N_{\text {initial }}=9$ gyrations, $N_{\text {design }}=135$ gyrations, and $\mathrm{N}_{\text {maximum }}=220$ gyrations, and these levels of gyration were used consistently in all sample preparations of SUPERPAVE trial mixes in the study. The initial trial asphalt binder content for the three blends was estimated to be $4.96 \%$. Two specimens from each trial blend were compacted using the SGC.

Table 4 shows the results for the tested samples, in addition to the required estimated properties (Voids in Mineral Aggregate, VMA; Voids fill with Asphalt, VFA, Theoretical maximum specific gravity, $\mathrm{Gmm}$ at $\mathrm{Ni}$, and dust proportion) at the estimated asphalt content, to achieve $4 \%$ air voids at $\mathrm{N}$ design.

A unique gradation characteristic for mixed aggregates for SUPERPAVE is suggested for the restricted zone (RZ) (see Figure 3) to prevent the rutting effect of SUPERPAVE mixes. SUPERPAVE mix design requires the gradation of the optimum mixed aggregate not passing through the RZ. In this study, the gradation 
was trialled until it was found to comply with the SUPERPAVE mix design criteria as shown in Figure 3.

However it should be noted that this result is relevant to this study only, and it cannot yet be generalised to, or serve as a recommendation for the Thailand mix design. Where SUPERPAVE is employed, it is necessary to re-determine the optimum mix aggregate gradation to comply with SUPERPAVE gradation specifications. In addition to this, in this study, the SUPERPAVE recommended asphalt content is $5.0 \%$, which is lower than the Marshall design recommended optimum asphalt content. Table 5 demonstrates the mix recipes of asphalt test samples in the study.

Table 4.

Properties of the SUPERPAVE mix trial for $4 \%$ air void at $\mathrm{N}_{\text {design }}$

\begin{tabular}{|c|c|c|c|c|c|c|c|c|c|}
\hline \multirow{2}{*}{$\begin{array}{l}\text { Initial } \\
{ }^{*} A C \%\end{array}$} & \multicolumn{9}{|c|}{ Estimated properties to achieve $4 \%$ air void at $\mathrm{N}_{\text {design }}$} \\
\hline & ${ }^{*} \mathrm{AC} \%$ & ${ }^{*} \mathrm{VMA} \%$ & ${ }^{\star}$ Spec. & *VFA\% & ${ }^{\star}$ Spec. & $\begin{array}{l}{ }^{*} \% \mathrm{Gmm} \\
@ \mathrm{Ni}\end{array}$ & ${ }^{*}$ Spec. & $\begin{array}{l}{ }^{*} \text { Dust } \\
\text { Prop. }\end{array}$ & ${ }^{\star}$ Spec. \\
\hline 4.96 & 5 & 14.3 & $\begin{array}{l}13 \\
\min \end{array}$ & 72.38 & $65-75$ & 86.3 & $\begin{array}{l}89 \\
\max .\end{array}$ & 0.91 & $0.6-1.2$ \\
\hline
\end{tabular}

${ }^{*}$ Note: $\mathrm{AC}=$ Asphalt Content; VMA $\%=\%$ Voids in the Mineral Aggregate; VFA\%=\%Voids filled with asphalt; \%Gmm@ Ni = A maximum percentage of the theoretical density (Gmm) requirement at Ni (initial number of gyrations); Spec. = Specification; Dust Prop.=Dust proportion

Table 5.

Details of the SUPERPAVE test samples and the Marshall test samples

\begin{tabular}{lcc}
\hline Details & SUPERPAVE samples & Marshall samples \\
\hline Aggregates & Crushed limestone with DOH spec [14] \\
Asphalt contents & $5.0 \%$ & $5.2 \%$ \\
Gyratory Compaction & 135 gyrations & 135 gyrations \\
\hline
\end{tabular}

\subsection{Performance evaluation of SUPERPAVE and Marshall mixes}

A comparison of the performances of both the Marshall and SUPERPAVE mix design procedures was made at this stage of the study. The test samples were prepared at the obtained optimum mix design asphalt contents for both procedures (i.e., AC 5.2\% for Marshall and AC 5.0\% for SUPERPAVE), using the gradation characteristics of local $\mathrm{DOH}$ specifications for the recommended gradation of the Marshall samples and the SUPERPAVE recommended gradation (see Figure 3) of the SUPERPAVE samples.

The compaction of test samples was performed using the gyratory compactor for SUPERPAVE and ASTM D1559, and using the 75-blow Marshall compactor for the Marshall procedure to achieve the targeted $4 \%$ air voids for both methods. The test samples were subjected to a comprehensive mechanical evaluation testing program, comprising Marshall stability, loss of Marshall stability, indirect tensile strength, loss of indirect tensile strength, resilient modulus, and dynamic creep performance 


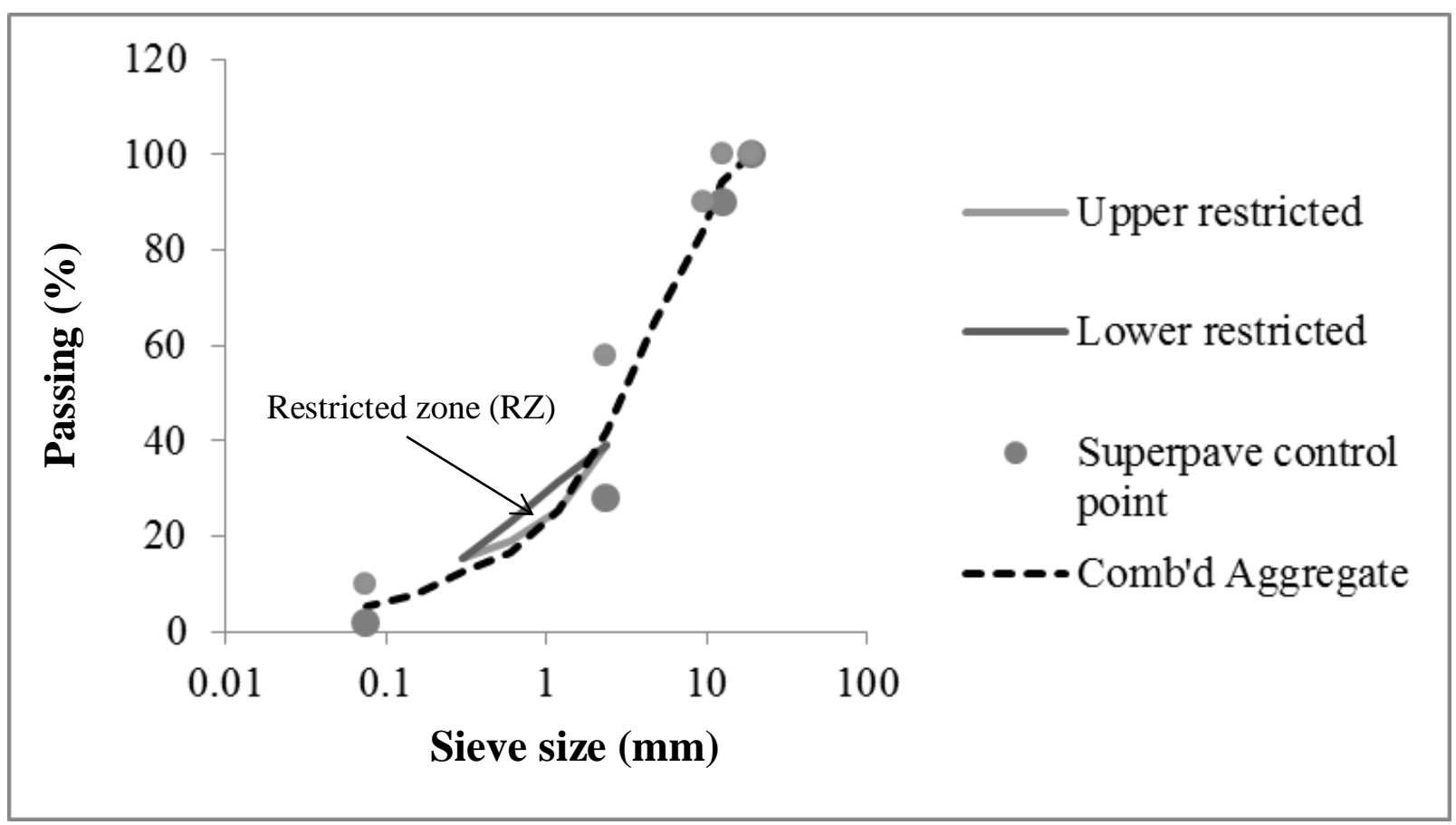

Figure 3. Gradation characteristics of the mixed aggregate used in this study

\subsubsection{Marshall stability and loss of Marshall stability (ASTM D1559)}

Six samples were prepared from each mix and immersed in a water bath at a temperature of $60^{\circ} \mathrm{C}$. After 30 minutes of immersion in the bath, the first three samples from each mix were tested for Marshall stability. The second three samples were tested for Marshall stability after 24 hours immersion.

Figure 4 shows the results for the tested samples. The results demonstrate that the SUPERPAVE bath samples show higher Marshall Stability than the Marshall samples, this being true for both the initial stability values and wet stability values. In addition, the SUPERPAVE samples demonstrated a lower level of loss of Marshall stability than the Marshall samples. The superiority of SUPERPAVE samples over Marshall samples would be attributable to the improved aggregate structure, lower asphalt content and lower dust proportion of the SUPERPAVE samples.

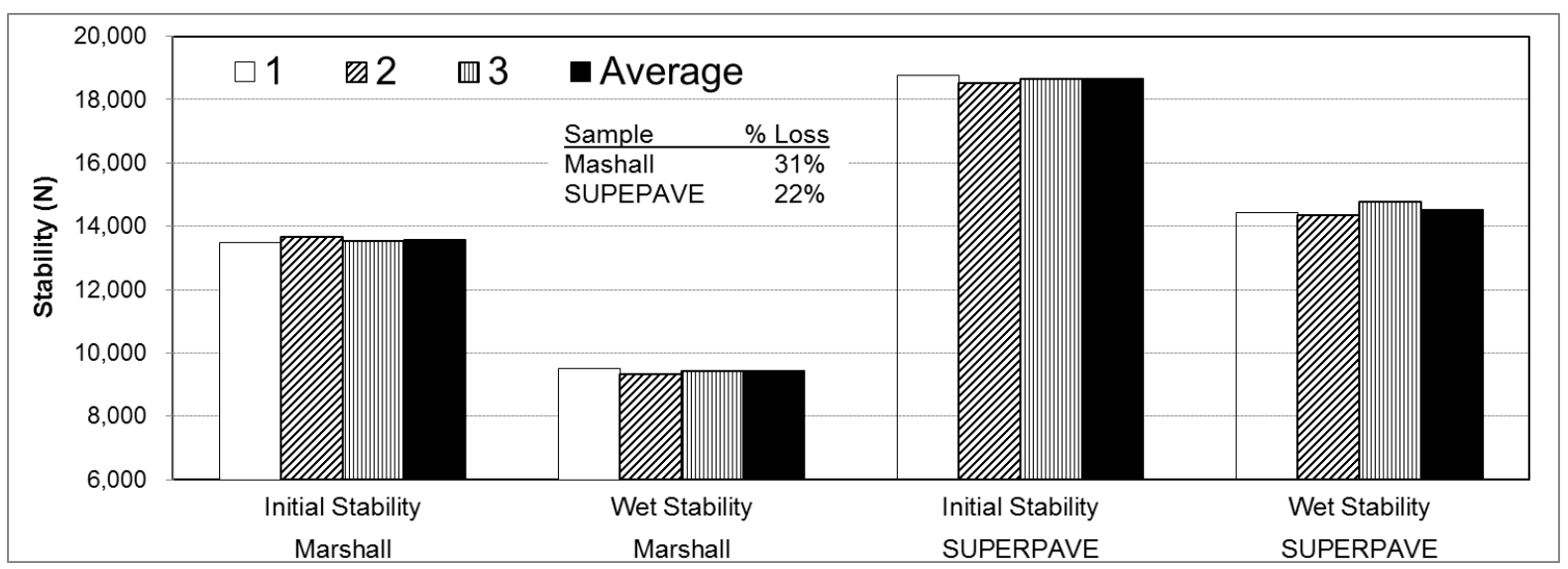


Figure 4. Marshall Stability test results

\subsubsection{Indirect tensile strength and loss of indirect tensile strength (AASHTO T-283)}

The Indirect Tensile Strength (ITS) value of an asphalt sample indicates the tensile resistance of an asphalt layer in a multi-layer system of road pavement. Furthermore, the stripping resistance (water susceptibility) of asphalt concrete mixes can be evaluated by the decrease in the loss of ITS after immersion in water for 24 hours at $60^{\circ} \mathrm{C}$, in accordance with the AASHTO T-283 test procedure [1] (Asi 2007) (Asi 2007).

Figure 5 illustrates the ITS and loss of ITS in this study. The results indicate that the average loss in strength due to water damage is lower in the SUPERPAVE samples than in the Marshall samples. Moreover, the ITS loss value for SUPERPAVE samples was $16 \%$, which is within the $20 \%$ allowable loss limit specified in the SUPERPAVE specifications [18]. In addition, Figure 5 indicates that both the SUPERPAVE ITS and wet ITS samples show higher ITS values than both Marshall samples.

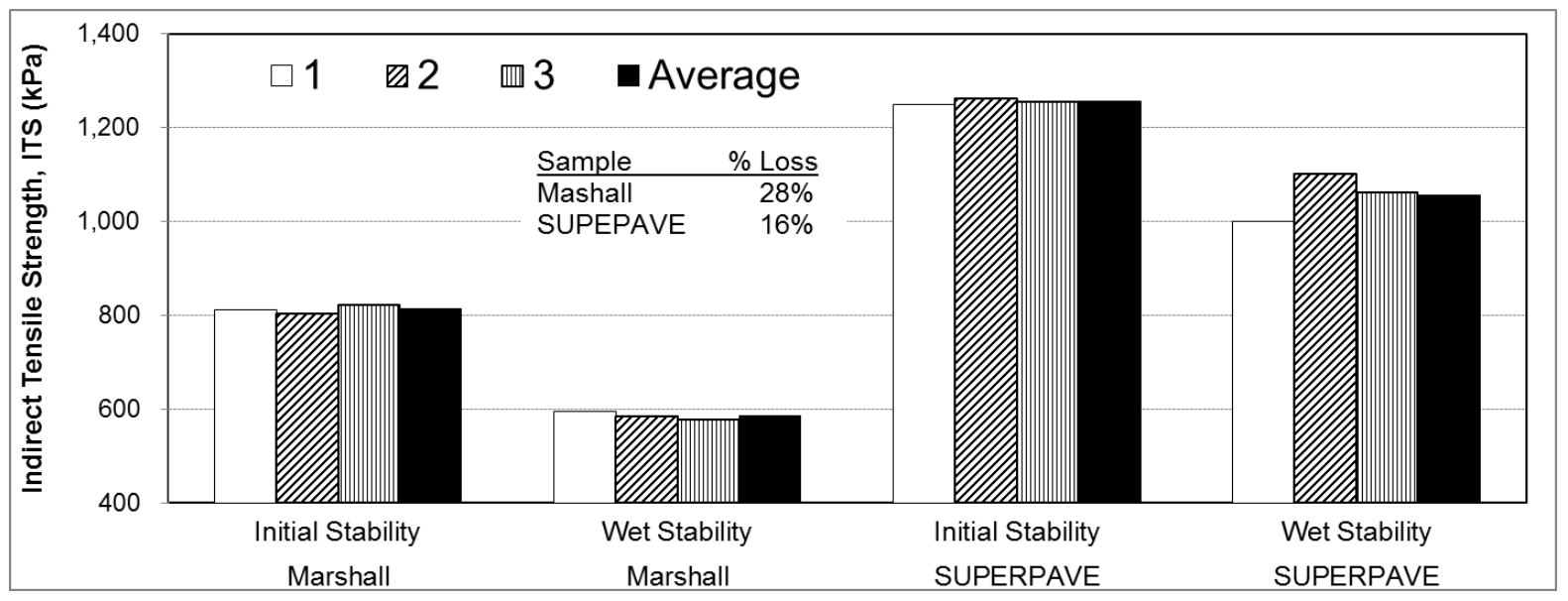

Figure 5. Indirect tensile strength test results

\subsubsection{Resilient modulus tests, MR (ASTM D 4123)}

Resilient modulus is the most important input parameter in the new mechanistic pavement structure design. It represents the cyclic pavement response in terms of dynamic stresses and their relation to recoverable strains. Three samples from each mix were placed in two positions for the diametrical Resilient Modulus (MR) test at $40^{\circ} \mathrm{C}$. Figure 6 shows the obtained MR values for all the tested mixes. It indicates that SUPERPAVE mixes have a higher diametric resilient modulus than Marshall mixes. 


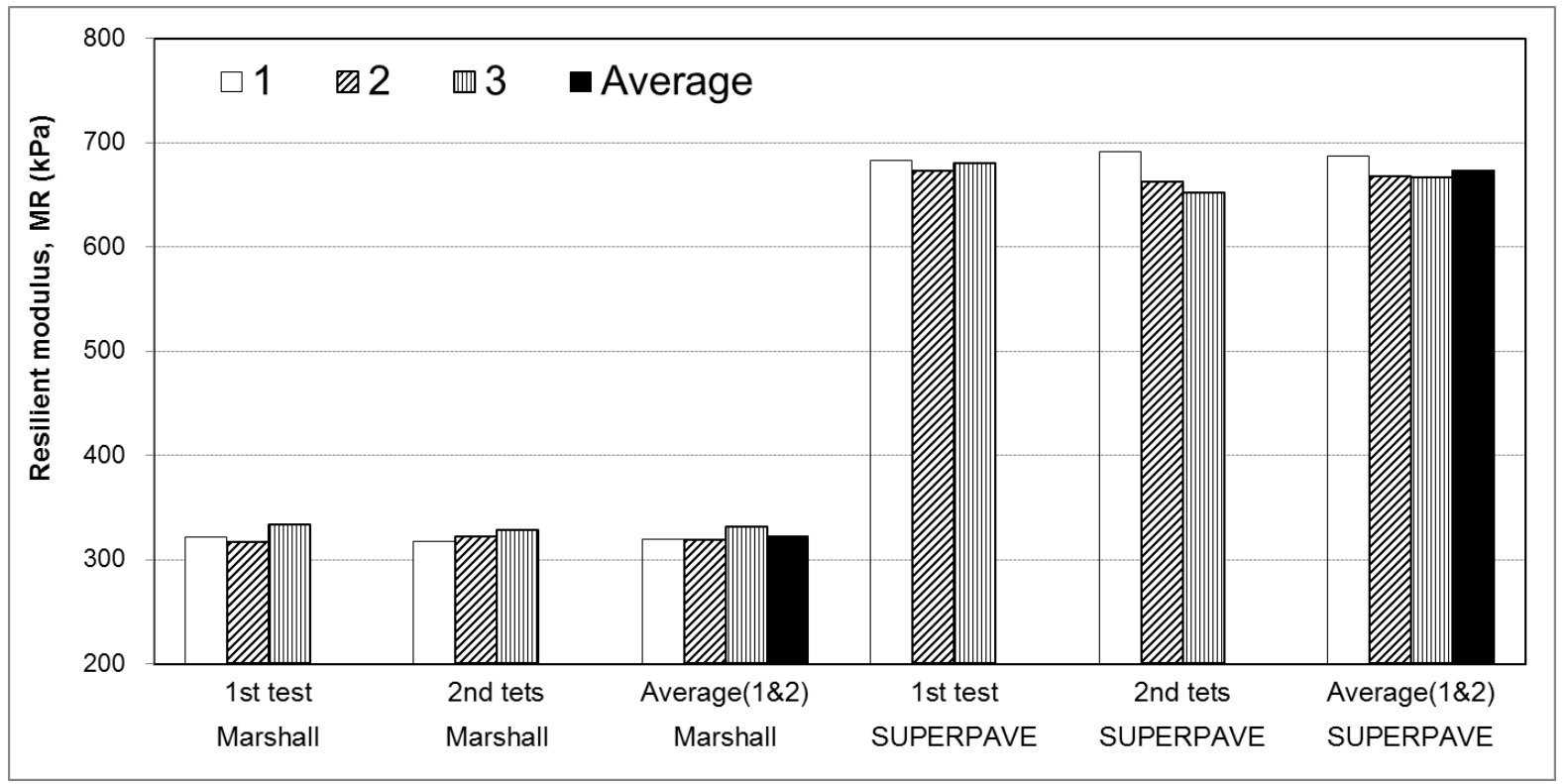

Figure 6. Resilient modulus test results

\subsubsection{Dynamic creep test (AS 2891.12.1)}

The dynamic creep test is used to evaluate the rutting potential of asphalt mixes [10]. It is suggested that rutting is a repeated creep mechanism developed under sinusoidal loading pulses [19]. The test is conducted by applying a static load to an asphalt specimen and measuring the resultant permanent deformation [10].

The dynamic creep specimen tests were performed in accordance with Australian Standard AS 2891.12.1 - 1995: Determination of permanent compressive strain characteristics of asphalt - Dynamic creep test. The asphalt specimen briquettes from the resilient modulus test were re-used to conduct the dynamic creep test. Table 6 shows the comparison of the dynamic creep test results for both mixes, and shows that all the values for SUPERPAVE mix samples were far superior to those of the Marshall Mix samples. 
Table 6.

Dynamic creep test results

\begin{tabular}{|c|c|c|c|c|c|c|c|}
\hline $\begin{array}{l}\text { Mix } \\
\text { Type }\end{array}$ & $\begin{array}{c}\text { Spec. } \\
\text { No. }\end{array}$ & $\begin{array}{l}\text { Comp. } \\
\text { Stress } \\
(\mathrm{kPa})\end{array}$ & $\begin{array}{c}\text { Cycles to } \\
\text { Acc. Strain } \\
\text { at } 0.01\end{array}$ & $\begin{array}{l}\text { Cycles to } \\
\text { Acc. Strain } \\
\text { at } 0.03\end{array}$ & $\begin{array}{c}\text { Min. } \\
\text { Slope } \\
\text { (um/m/c) }\end{array}$ & $\begin{array}{c}\text { Acc. } \\
\text { Strain at } \\
\text { min. } \\
\text { slope } \\
(u m / m / c)\end{array}$ & $\begin{array}{l}\text { Test } \\
\text { Temp. } \\
\left({ }^{\circ} \mathrm{C}\right)\end{array}$ \\
\hline \multirow{3}{*}{$\begin{array}{l}\overline{\overline{0}} \\
\frac{0}{00} \\
\stackrel{0}{0} \\
\sum\end{array}$} & 1 & 201.6 & 912 & 4930 & 3.611 & 20366 & 50.0 \\
\hline & 2 & 197.7 & 933 & 4095 & 3.638 & 12368 & 49.9 \\
\hline & 3 & 199.9 & 1056 & 4458 & 3.526 & 14950 & 50.4 \\
\hline \multirow{3}{*}{ 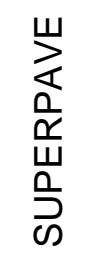 } & 1 & 202.4 & 1170 & 6205 & 2.917 & 22457 & 50.1 \\
\hline & 2 & 199.4 & 1492 & 3835 & 2.888 & 14586 & 49.9 \\
\hline & 3 & 198.7 & 1691 & 5774 & 2.821 & 12129 & 50.0 \\
\hline
\end{tabular}

\section{Conclusions}

This study was conducted to evaluate the possibility of implementing Superior Performance asphalt Pavement (SUPERPAVE) mixture specifications in Thailand with adjustments for compatibility with locally specific materials, traffic and environmental conditions. Based on the laboratory results of the temperature study, the performance of the designed asphalt mixes using both the Marshall and SUPERPAVE mix design procedures were evaluated and compared. The main conclusions to be drawn are as follows:

- The Performance Grade (PG grade) of the commonly-used asphalt binder in Thailand, AC 60-70, is PG 64-22 based on the performance grade of the used asphalt cement according to SHRP binder performance specification (AASHTO MP1), where DSR testing of fresh and treated samples was conducted at different test temperatures after the RTFO and Pressure PAV processes.

- The temperature zoning map produced for the study area, the North of Thailand, resulted in the development of two general grade zones of PG 6410 for one province and PG 70-10 for the rest of the area, for the minimum requirement of light traffic (less than 10 million ESALs). For more severe conditions of temperature and traffic higher PG numbers are required.

- Based on the performance grade of the Thailand asphalt derived from the experimental results and those derived from the temperature study, the commonly-used asphalt binders in Thailand (i.e., AC 60-70) cannot be used without the need for modification in all parts of the study area: the grading 
requires the shifting up of levels by one or two PG grades. In practice, superior quality asphalt binders and/or polymer modification techniques would be required.

- The local aggregate used in this study meets both SUPERPAVE consensus properties and source properties.

- The asphalt binder content determined from the SUPEPAVE mix design procedure is lower than that predicted by the Marshall Mix design procedure.

- SUPERPAVE mixes showed far superior performance to Marshall mixes based on all types of testing in this study.

- This study's findings cannot necessarily be applied to all commonly-used asphalt binders in the Thailand road network following the SUPERPAVE specifications; some adjustments are necessary. Despite these adjustments, the SUPERPAVE mixture specifications prove far superior to those of the Marshall Mix design procedure.

Therefore it is the recommendation of this study that the SUPERPAVE mix design procedure be adopted (with adjustments as required) to replace the Marshall Mix design procedure in Thailand.

\section{Acknowledgements}

The authors wish to express their gratitude to the Thailand Research Fund (TRF) scheme "TRF Research Career Development Grant (2008-2010)" for their financial support in this project (MRG5280105). Special thanks are extended to the North Thailand Meteorological Centre for providing weather data information. In addition the team would like to extend their appreciation to Dr. Tunwin Svasdisant of the Department of Highway, Thailand, and Curtin University, Australia as the mentor of this project for their valuable laboratory and technical support.

\section{References}

[1] Asi IM. Performance evaluation of SUPERPAVE and Marshall asphalt mix designs to suit Jordan climatic and traffic conditions. Construction and Building Materials 2007;21:1732-40.

[2] Bahia HU. Bibliographies for physical properties of asphalt cement. SHRP-A-626,National Research Council, Washington, DC.

[3] American Society for Testing and Materials (ASTM). Standard test methods, vol. 4.03. West Conshohocken, PA: ASTM; 1997.

[4] Ubstutye A. Mix design methods for asphalt concrete and other hot-mix types Manual Series No.2 (MS-2), Asphalt Institute; 1993.

[5] Svasdisant T, Kortangsampant T. Guideline for asphalt cement selection and asphalt mix design to suit Thailand conditions, Department of Highways, Kingdom of Thailand; 2010.

[6] Asphalt Institute. Performance graded asphalt binder specification and testing, Superpave Mix Design Series No. 1 (SP-1), Asphalt Institute Research Center, Lexington, KY; 1997.

[7] Asphalt Institute. Superpave Mix Design Series No. 2 (SP-2), Asphalt Institute Research Center, Lexington, KY; 2001.

[8] Roberts F, Mohammad M, Wang L. History of hot mix asphalt mixture design in the United States. J Mater Civil Eng 2002;14(4):279-93. 
[9] American Society for Testing and Materials (ASTM). Standard test methods, vol. 4.03. West Conshohocken, PA: ASTM; 2000.

[10] Brown R, Kandhal P, Zhang J. Performance testing for hot mix asphalt. National Center for Asphalt Technology, Report No. 2001-05A, Auburn University, Alabama; 2001.

[11] Jitsangiam P. An evaluation of the suitability of SUPERPAVE and Marshall asphalt mix designs related to Thailand's climatic conditions; 2010. Grant No. MRG5280105. Sponsored by Thailand Research Fund. Kingdom of Thailand.

[12] Federal Highway Administration (FHWA). Background of SUPERPAVE asphalt mixture design and analysis. Publication No. FHWA-SA-95-003, US Department of Transportation, Washington, DC; 1995.

[13] Anderson RM. SUPERPAVE level 1 mixture design example. A first look at volumetric mix design in the SUPERPAVE system. Lexington, KY: Asphalt Institute Research Center; 1993.

[14] Department of Highways (DOH). Standard No. DH-S 408/1989, Kingdom of Thailand; 1989.

[15] Department of Highways (DOH). Specification No. DH-SP. 401/1988, Kingdom of Thailand; 1988.

[16] Federal Highway Administration (FHWA). LTPPbind software [Computer program]. US Department of Transportation, Washington, DC; 1999.

[17] Department of Highways (DOH). Standard No. DH-T 604/1974, Kingdom of Thailand; 1974.

[18] Cominsky RJ, Huber GA, Kennedy TW, Anderson M. The Superpave mix design manual for new construction and overlay. SHRP-A-407, National Research Council, Washington, DC; 1994.

[19] Minnesota Department of Transportation. Inventory of properties of Minnnesota certified asphalt binders. Final report. 2004Apr. Report No.MN/RC-2004-35. Contract No.: (c)81655 (wo)64. 